\title{
Light Localization in Left-Handed Media
}

\author{
M. Rusek, A. Oreowski And J. Mostowski \\ Institute of Physics, Polish Academy of Sciences \\ al. Lotników 32/46, 02-668 Warsaw, Poland
}

\begin{abstract}
Localized waves in disordered left-handed materials are studied using a generalized coupled-dipole model. Resonances in an open system consisting of randomly distributed electric and magnetic dipoles are investigated. A new type of long-lived resonance modes localized at the boundary of the system is found. They resemble evanescent waves responsible for a superfocusing phenomenon by a left-handed lens.
\end{abstract}

PACS numbers: 03.65.Nk, 41.20.Jb, 72.10.Fk, 72.15.Rn

\section{Introduction}

Recently artificially built materials (metamaterials) with negative electric and magnetic permeability have attracted much attention (see [1] and references therein). The directions of phase and energy propagation in these so-called left-handed media are opposite. Thus the wave vector and the electric and magnetic fields vectors form a left-handed set. The refraction index in such media is negative and a wave incident on a left-handed medium is refracted in an opposite direction.

Usually left-handed materials are built of artificial electric and magnetic resonators with specially tailored frequency response. The system of electric resonators (wires) and magnetic resonators (split ring resonators) forms a periodic lattice. Such a metamaterial works as a left-handed medium for electromagnetic waves with frequencies from a narrow window only.

In this paper we investigate a left-handed medium built of randomly placed scattering elements. A motivation behind such a random arrangement of scatterers is that it may make the properties of the medium less sensitive on the properties of the individual scatterers. This can result in a wider frequency window in which left-handed medium works.

This idea is based on the observations from solid state physics. In crystalline solids a narrow forbidden band gap exists. No propagating modes exist in this energy region. By introducing disorder into the system the gap becomes wider but it is no longer a strictly forbidden gap. Some propagating modes are allowed. However, for sufficiently large disorder no propagating modes are allowed again in an energy band given by the Ioffe-Regel criterion. Instead a band of localized 
states appears. The width of this band is usually much larger than the width of the original forbidden band gap.

Localized waves are orthogonal to all propagating waves (plane waves). Thus they have to be composed of evanescent waves only [2]. Recently focusing of electromagnetic waves by left-handed materials has attracted much attention [3-6]. It was suggested that "evanescent" waves in such a medium are in fact not evanescent at all: instead of decaying, their amplitude should actually grow up as they pass through a left-handed material. This unusual behavior of evanescent waves may modify the properties of localized waves. Thus a study of the Anderson localization in disordered left-handed materials seems interesting.

This paper is organized as follows. In Sect. 2 the discrete dipole model of left-handed media is introduced. In Sect. 3 it is used to model a left-handed lens. It is shown that a random collection of dipoles with suitably chosen scattering properties does act as a left-handed medium. The disordered system studied in this section is in the localization regime as given by the Ioffe-Regel criterion. Long-lived resonance states in such a system may form a band of localized waves in the limit of an infinite system. Example localized resonance modes are studied in Sect. 4. We finish with some conclusions in Sect. 5.

\section{Discrete-dipole model of left-handed media}

The left-handed medium under consideration is modeled using a generalized discrete-dipole approach. In a standard discrete-dipole approach a dielectric medium is modeled by a system of interacting electric dipoles [7]. A generalization employed in this paper consists of simultaneous consideration of both electric and magnetic dipoles. This is necessary to model a left-handed medium.

For simplicity the electric and magnetic dipoles will be placed at the same points. Thus the polarization $\boldsymbol{P}$ and magnetization $\boldsymbol{M}$ of the medium read as

$$
\begin{aligned}
& \boldsymbol{P}(\boldsymbol{r})=\sum_{a} \boldsymbol{p}_{a} \delta\left(\boldsymbol{r}-\boldsymbol{r}_{a}\right), \\
& \boldsymbol{M}(\boldsymbol{r})=\sum_{a} \boldsymbol{m}_{a} \delta\left(\boldsymbol{r}-\boldsymbol{r}_{a}\right) .
\end{aligned}
$$

The positions of the dipoles $\boldsymbol{r}_{a}$ are the same in both equations.

In the standard discrete dipole approach an electric moment $\boldsymbol{p}_{a}$ of the $a$-th dipole is coupled to the electric field $\boldsymbol{E}^{\prime}$ by a microscopic electric polarizability $\alpha$ :

$$
\boldsymbol{p}_{a}=\frac{\alpha}{1-\frac{2}{3} \mathrm{i} k^{3} \alpha} \boldsymbol{E}^{\prime}\left(\boldsymbol{r}_{a}\right),
$$

where $k$ is the wave number in vacuum. The microscopic electric polarizability $\alpha$ is related to the macroscopic dielectric permeability $\epsilon$ by the Clausius-Mosotti formula

$$
\alpha=\frac{3}{4 \pi n} \frac{\epsilon-1}{\epsilon+1},
$$

where $n$ is the density of the dipoles. Equation (4) is valid if the density of the 
dipoles per wavelength cubed is large enough. In the limit of an infinite density the discrete system of dipoles (1) is equivalent to a homogeneous dielectric medium.

According to the Clausius-Mosotti formula (4) the product of the density and polarizability is a constant. Thus in the limit of large density of dipoles the mean free path $l \propto 1 /\left(n \alpha^{2}\right)$ becomes much larger than the wavelength. Therefore, the Ioffe-Regel criterion is no longer fulfilled and the system is out of localized regime. In our calculations we will be interested in intermediate densities: sufficiently large to be able to reasonably model a homogeneous dielectric and sufficiently low to have the mean free path lower than the wavelength.

In our model Eqs. (3) and (4) are supplemented by analogous relations for magnetic dipole moment

$$
\boldsymbol{m}_{a}=\frac{\beta}{1-\frac{2}{3} \mathrm{i} k^{3} \beta} \boldsymbol{H}^{\prime}\left(\boldsymbol{r}_{a}\right)
$$

and microscopic magnetic polarizability and macroscopic magnetic permeability

$$
\beta=\frac{3}{4 \pi n} \frac{\mu-1}{\mu+1} .
$$

The electric field acting on the electric moment of the $a$-th dipole from Eq. (3) is the sum of the incident field and waves scattered by all other dipoles

$$
\boldsymbol{E}^{\prime}\left(\boldsymbol{r}_{a}\right)=\boldsymbol{E}_{0}\left(\boldsymbol{r}_{a}\right)+\sum_{a \neq b} \boldsymbol{E}_{b}\left(\boldsymbol{r}_{a}\right)
$$

Similarly the magnetic field acting on the magnetic moment of the $a$-th dipole is given by

$$
\boldsymbol{H}^{\prime}\left(\boldsymbol{r}_{a}\right)=\boldsymbol{H}_{0}\left(\boldsymbol{r}_{a}\right)+\sum_{a \neq b} \boldsymbol{H}_{b}\left(\boldsymbol{r}_{a}\right)
$$

Electric and magnetic fields radiated by an $a$-th dipole can be expressed in terms of Green's tensors $\hat{G}_{1}$ and $\hat{G}_{2}$ (for explicit forms of these tensors see, e.g., [8]):

$$
\begin{aligned}
& \boldsymbol{E}_{a}(\boldsymbol{r})=\hat{G}_{1}\left(\boldsymbol{r}-\boldsymbol{r}_{a}\right) \cdot \boldsymbol{p}_{a}-\hat{G}_{2}\left(\boldsymbol{r}-\boldsymbol{r}_{a}\right) \cdot \boldsymbol{m}_{a}, \\
& \boldsymbol{H}_{a}(\boldsymbol{r})=\hat{G}_{2}\left(\boldsymbol{r}-\boldsymbol{r}_{a}\right) \cdot \boldsymbol{p}_{a}+\hat{G}_{1}\left(\boldsymbol{r}-\boldsymbol{r}_{a}\right) \cdot \boldsymbol{m}_{a} .
\end{aligned}
$$

It is seen from Eqs. (9) that the electric field radiated by magnetic dipoles acts on electric dipoles, and the magnetic field radiated by electric dipoles acts on magnetic dipoles. Of course, the dipoles of the same kind keep interacting with one another by a respective field as well.

\section{New type of lens}

A slab made of a left-handed material can act as a new type of lens [3]. A particular interest has been paid to the case of

$$
\epsilon=-1, \quad \mu=-1
$$

where there is no reflection at the slab boundaries, only refraction.

We investigate this situation by simulating numerically a left-handed lens built up of a collection of 1000 dipoles. Their random distribution is depicted in Fig. 1. The dipoles form a slab of thickness $d=1$ wavelength. The density of the 


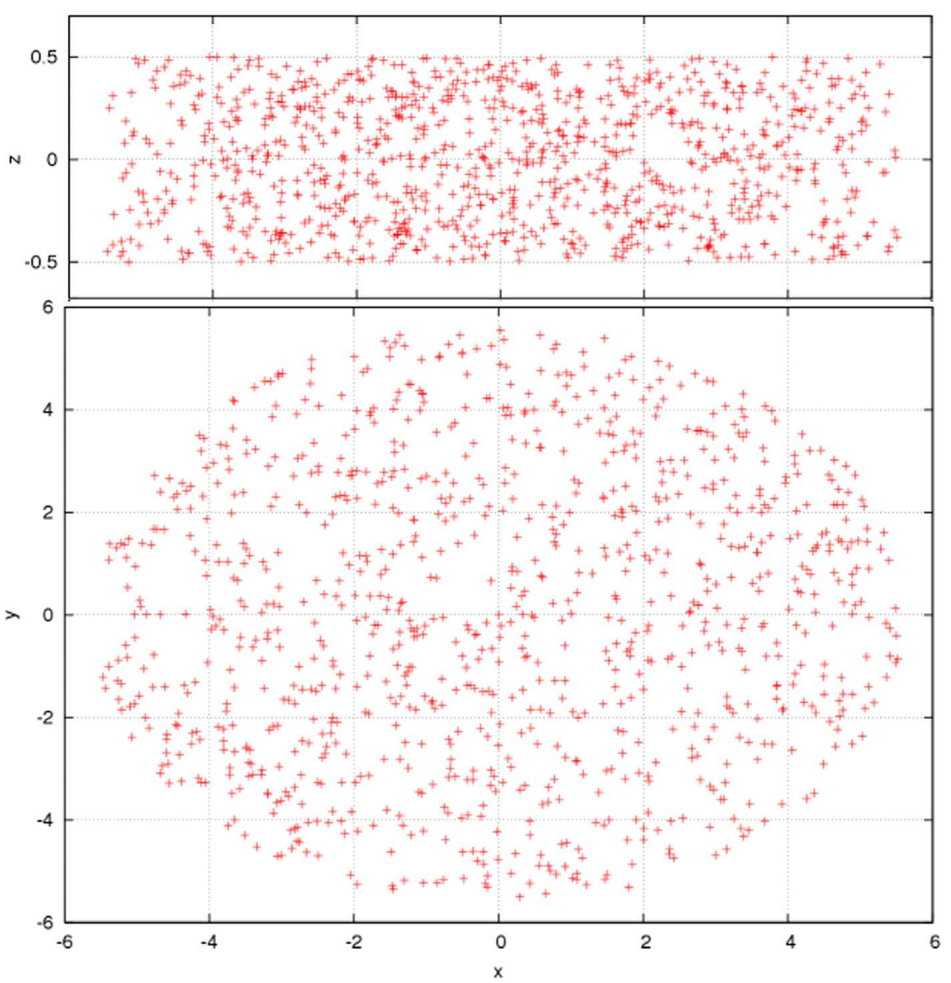

Fig. 1. Flat cylinder made of randomly distributed dipoles; side view (left) and bottom view (right). All dimensions are scaled in wavelength.

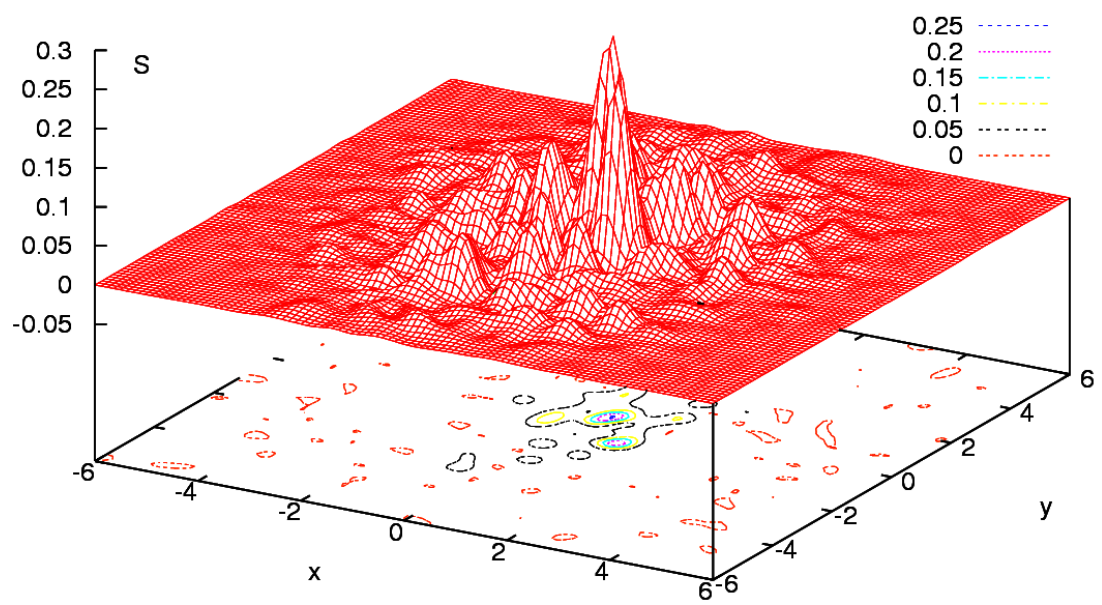

Fig. 2. Image of a point dipole formed by the left-handed lens from Fig. 1. 
dipoles is $n=10$ dipoles per wavelength cubed. Their polarizabilities $\alpha$ and $\beta$ are calculated according to Eqs. (4) and (6) in order to reproduce Eq. (11).

The slab built of passive dipoles is illuminated by an additional active electric dipole placed at point $x_{0}=0, y_{0}=0, z_{0}=-1$. Its dipole moment $\boldsymbol{p}_{0}$ is given and it is the source of fields $\boldsymbol{E}_{0}$ and $\boldsymbol{H}_{0}$ from Eqs. (7) and (8).

This field calculated according to Eqs. (9) at the positions of the remaining dipoles $\left(\boldsymbol{E}_{0}\left(\boldsymbol{r}_{a}\right)\right.$ and $\left.\boldsymbol{H}_{0}\left(\boldsymbol{r}_{a}\right)\right)$ is substituted into the system of linear Eqs. (3), (5), (7)-(9), which gives us the remaining dipole moments $\boldsymbol{p}_{a}$ and $\boldsymbol{m}_{a}(a=1, \ldots, 1000)$.

Then all the dipole moments $\boldsymbol{p}_{a}$ and $\boldsymbol{m}_{a}(a=0, \ldots, 1000)$ are substituted again into Eqs. (9) to calculate the field on the screen. The screen is a planar surface parallel to the bottom of the cylinder from Fig. 1 and described by the equation $z=z_{s}$. The equation of a left-handed lens [3] gives

$$
z_{s}-z_{0}=d \text {. }
$$

The time averaged field energy stream through the surface of the screen (given by the $z$-th component of the Poynting vector) is depicted in Fig. 2. We see that the image of the point source $\boldsymbol{p}_{0}$ is indeed visible on the screen. This proves that the system of dipoles from Fig. 1 indeed acts as a left-handed lens.

\section{Resonances in a random system of dipoles}

According to theory of disordered media in the limit of an infinite system a band of localized modes may appear. This so-called Anderson localization occurs if the medium is sufficiently disordered. A common criterion for critical disorder was given by Ioffe and Regel, i.e., the mean free path $l$ in the system needs to be smaller than the wavelength

$$
k l<1 \text {. }
$$

For the random collection of dipoles from Sect. 3 we have $k l \approx 0.66$. Thus the IoffeRegel criterion (13) is satisfied and this disordered system is in the localization regime. In an open system localized modes become long-lived resonances.

A way of dealing with those resonances is to look for resonance poles in the complex frequency plane. Approximate frequencies and widths of the resonances can be obtained by diagonalizing the matrix of the system of linear Eqs. (3), (5), (7)-(9). In order to be able to interpret the eigenvalues as the resonance poles we need to introduce a model of scattering of a single dipole $[2,9]$.

Let us consider a simple scatterer with one internal resonance. The scattering cross-section has a form

$$
k^{2} \sigma=12 \pi \frac{\gamma_{0}^{2}}{\left(\omega-\omega_{0}\right)^{2}+\gamma_{0}^{2}} .
$$

To model this so-called Breit-Wigner scatterer the polarizability $\alpha$ from Eq. (3) needs to be defined as follows:

$$
\frac{2}{3} k^{3} \alpha=-\frac{\gamma_{0}}{\omega-\omega_{0}} .
$$

The same holds for $\beta$ from Eq. (5). 
Let us note that the scattering cross-section is twice larger than a cross-section corresponding to electrical dipole only. Thus in a system of electric and magnetic dipoles localization should happen at density twice as low than reported previously for a system of electrical dipoles [10].

It is a common believe that resonance modes corresponding to long-lived resonances are well localized inside the system. Thus the adding of additional dipoles at a system boundary should not affect their shape significantly and in the limit of the infinite system they will become localized waves. As will be seen from the subsequent considerations it does not need to be the case for left-handed media.

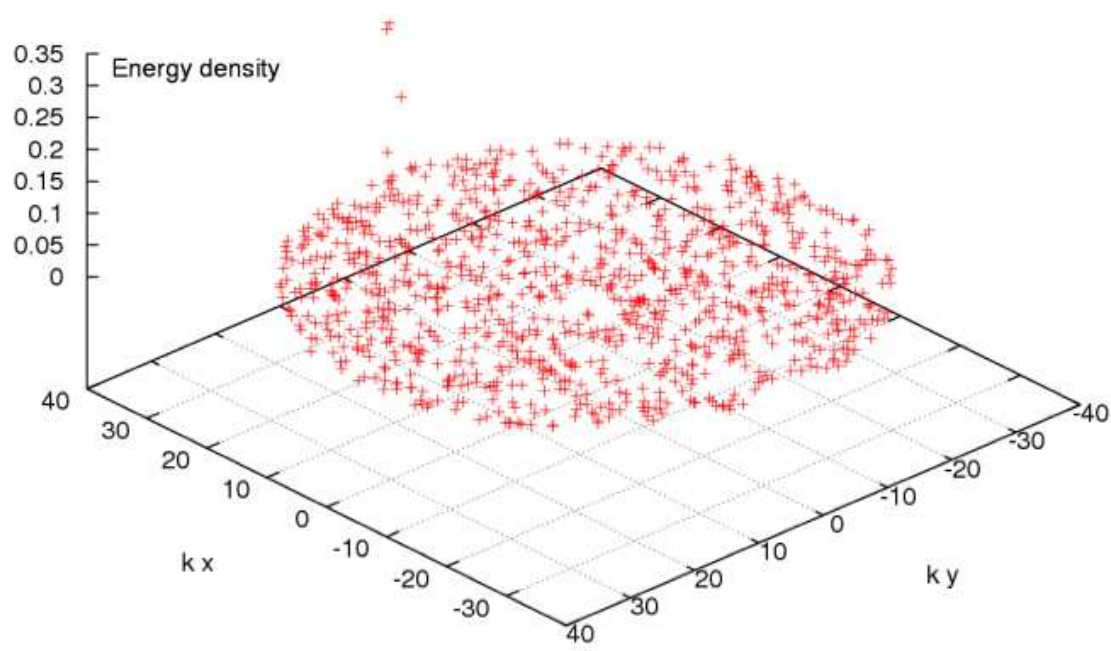

Fig. 3. Field energy density at the dipoles versus the dipole positions projected on the $x y$ plane. Six dipoles are excited in this mode.

Having solved the relevant equations we can search for the long-lived resonances. It happens that the longest-lived one is associated with a proximity resonance between pair of dipoles close to each other (see, e.g., [9]). Surprisingly enough, after eliminating all such cases, there still are interesting very long-lived resonances created by several dipoles. To prove it is not a proximity resonance we provide Fig. 3 showing the field energy density at the dipole positions $W_{a}$ versus the dipole positions $\boldsymbol{r}_{a}$ :

$$
W_{a}=\left|E^{\prime}\left(\boldsymbol{r}_{a}\right)\right|^{2}+\left|H^{\prime}\left(\boldsymbol{r}_{a}\right)\right|^{2} .
$$

It is clearly seen that several dipoles are excited.

In Fig. 4 we show a smoothed surface plot corresponding to the above discrete function. It is composed of evanescent modes and is evidently well localized but what is very interesting it is located (in one dimension) at the surface of our lens! We find this observation very exciting and plan to pursuit further investi- 

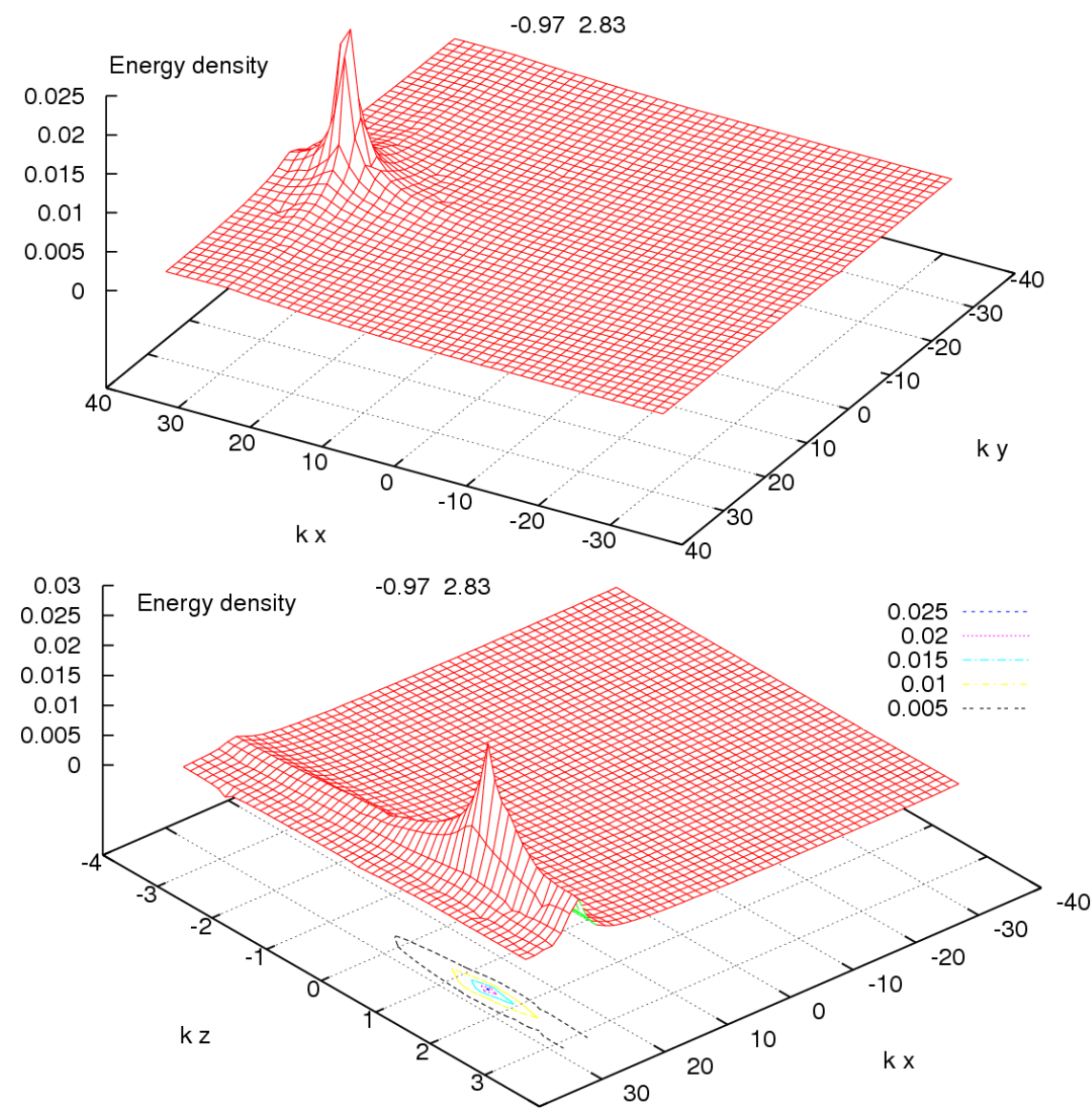

Fig. 4. Surface plot corresponding to Fig. 3.

gations along these lines. Here let us only remark that despite being composed of evanescent waves it does couple to the field radiated by our source dipole. The reason is actually very simple: source dipole is close enough to the lens to make also its near field essential.

The numbers visible in Fig. 4 describe the width and frequency of this resonance mode

$$
\begin{aligned}
& \frac{\gamma-\gamma_{0}}{\gamma_{0}}=-0.98, \\
& \frac{\omega-\omega_{0}}{\gamma_{0}}=2.84 .
\end{aligned}
$$

Thus the width of this resonance $\gamma$ is 200 smaller than the width of the single scatterer resonance $\gamma_{0}$. 


\section{Brief summary}

A generalized coupled-dipole model, considering both electric and magnetic dipoles, has been developed and applied to study localized modes in left-handed media. Density of the dipoles is such that the model well approximates a continuous left-handed material and, simultaneously, the Ioffe-Regel criterion for onset of the Anderson localization is satisfied. We have discovered an interesting phenomenon of a new type of collective localized long-lived resonances (not related to proximity resonances) located close to the boundary of the medium. It is apparently not the case for ordinary disordered dielectric media. There is plenty of such modes but if they form a band of localized waves with growing size of the medium is an open question. This nontrivial observation will be explored in more detail in a forthcoming paper.

\section{References}

[1] Metamaterials. Physics and Engineering Explorations, Eds. N. Engheta, R.W. Ziolkowski, Wiley-IEEE Press, 2006.

[2] M. Rusek, A. Orłowski, Phys. Rev. E 59, 3655 (1999).

[3] J.B. Pendry, Phys. Rev. Lett. 85, 3966 (2000).

[4] G.W. 't Hooft, Phys. Rev. Lett. 87, 249701 (2001).

[5] J.M. Williams, Phys. Rev. Lett. 87, 249703 (2001).

[6] M. Nieto-Vesperinas, N. Garcia, Phys. Rev. Lett. 91, 099702 (2003).

[7] B.T. Draine, P.J. Flatau, J. Opt. Soc. Am. A 11, 1491 (1994).

[8] J.D. Jackson, Classical Electrodynamics, Wiley, New York 1962.

[9] M. Rusek, J. Mostowski, A. Orłowski, Phys. Rev. A 61, 022704 (2000).

[10] F.A. Pinheiro, M. Rusek, A. Orlowski, B.A. van Tiggelen, Phys. Rev. E 69, 026605 (2004). 